\title{
Strong evidence for an accelerating Universe
}

\author{
Balakrishna S. Haridasu ${ }^{1}$, Vladimir V. Luković ${ }^{2,3}$, Rocco D'Agostino ${ }^{2,3}$, and Nicola Vittorio ${ }^{2,3}$ \\ ${ }^{1}$ Gran Sasso Science Institute (INFN), Viale Francesco Crispi 7, 67100 L'Aquila, Italy \\ e-mail: sandeep.haridasu@gssi.infn.it \\ 2 Dipartimento di Fisica, Università di Roma "Tor Vergata", via della Ricerca Scientifica 1, 00133 Roma, Italy \\ 3 Sezione INFN, Università di Roma "Tor Vergata", via della Ricerca Scientifica 1, 00133 Roma, Italy
}

Received 19 January 2017 / Accepted 22 February 2017

\begin{abstract}
A recent analysis of supernova Ia (SN Ia) data claims a "marginal" $(\sim 3 \sigma)$ evidence for a cosmic acceleration. This result has been complemented with a non-accelerating $R_{\mathrm{h}}=c t$ cosmology, which was presented as a valid alternative to the $\Lambda$ CDM model. In this paper we use the same analysis to show that non-marginal evidence for acceleration is truly found. We compare the standard Friedmann models to the $R_{\mathrm{h}}=c t$ cosmology by complementing SN Ia data with baryon acoustic oscillations, gamma ray bursts, and observational Hubble datasets. We also study the power-law model, which is a functional generalisation of $R_{\mathrm{h}}=c t$. We find that the evidence for late-time acceleration cannot be refuted at a $4.56 \sigma$ confidence level from SN Ia data alone, and at an even stronger confidence level $(5.38 \sigma)$ from our joint analysis. In addition, the non-accelerating $R_{\mathrm{h}}=c t$ model fails to statistically compare with the $\Lambda \mathrm{CDM}$, having a $\triangle$ (AIC) $\sim 30$.
\end{abstract}

Key words. cosmology: observations - dark energy - cosmological parameters

\section{Introduction}

The very first evidence for an accelerated expansion of the Universe was obtained using the supernova Ia (SN Ia) observations in Riess et al. (1998) and Perlmutter et al. (1999), which has been further confirmed with the most recent supernova data (Betoule et al. 2014). Other low-redshift data such as the observational Hubble parameter (OHD, Jimenez \& Loeb 2002), baryon acoustic oscillations (BAO, Eisenstein et al. 2005) also support an accelerating Universe. As an independent observation, the cosmic microwave background (CMB) radiation has agreed excellently with these results and has provided the most stringent constraints on the cosmological models (Ade et al. 2016). These observations have established the $\Lambda \mathrm{CDM}$ as the concordance model of cosmology, and the late-time acceleration has been a widely accepted phenomenon.

However, Nielsen et al. (2016) have used a modified statistical model for the analysis of the supernova JLA dataset (Betoule et al. 2014) and claimed that the evidence for the acceleration is marginal $(\$ 3 \sigma)$. The modification was made by assuming an intrinsic variation in the $\mathrm{SN}$ absolute magnitude and in the light curve (colour and stretch) corrections, which were modelled as Gaussian. More recently, Rubin \& Hayden (2016) have strongly criticised this approach as incomplete and suggested using redshift dependent ad hoc functions for these corrections, presenting the evidence for acceleration to be $\sim 4.2 \sigma$. In this paper we want to show that even with the less flexible modelling of Nielsen et al. (2016) the evidence for acceleration is very strong. We also extend the joint analysis done in Luković et al. (2016) with the inclusion of gamma-ray bursts (GRB) dataset (Wei 2010).

The marginal evidence for an accelerating universe quoted in Nielsen et al. (2016) implies a scenario with very low dark matter and dark energy densities. As this scenario converges towards the Milne model, it has been complemented with the $R_{\mathrm{h}}=c t$ cosmology (Melia \& Shevchuk 2012; Melia 2012b,a), which features a non-accelerating linear expansion of the universe. This model essentially advocates that the Hubble sphere is same as the particle horizon of the universe (Bikwa et al. 2012). Also, the $R_{\mathrm{h}}=c t$ model has often been regarded as a Milne universe (Mitra 2014; Bilicki \& Seikel 2012) and several physical issues against this interpretation have been raised (Lewis 2013; Lewis \& van Oirschot 2012). In fact, this model preserves the linear expansion by imposing the constraint on the total equation of state $(\mathrm{EoS})\left(\rho_{\mathrm{tot}}+3 p_{\mathrm{tot}}=0\right)$, without requiring $\rho_{\mathrm{tot}}=p_{\mathrm{tot}}=0$. It is interesting to note that this model also coincides with the linear coasting models that are discussed in the context of modified gravity (Gehlaut et al. 2003; Dev et al. 2001, 2002). In a recent work, Kumar (2016) presented a linear coasting model in $f(R)$ gravity, which is functionally equivalent to the $R_{\mathrm{h}}=c t$ model. A linear expansion model can be generalised to a power-law model with an exponent $n$, which was brought up as an alternative to the standard model as it does not have the flatness and horizon problems (Sethi et al. 2005). In addition, it has been shown in Dolgov (1997) that classical fields coupling to spacetime curvature can give rise to a back-reaction from singularities, which can change the nature of expansion from exponential to power-law.

The $R_{\mathrm{h}}=c t$ model has been tested time and again, and contradictory conclusions have been presented. Bilicki \& Seikel (2012) have pointed out some of the problems in this model using the cosmic chronometer data from Moresco et al. (2012b,a) and radial BAO measurements, along with several modelindependent diagnostics, to show that $R_{\mathrm{h}}=c t$ is not a viable model. In Melia (2015) several claims against $R_{\mathrm{h}}=c t$ were refuted, and a list of works favouring $R_{\mathrm{h}}=c t$ over $\Lambda \mathrm{CDM}$ was compiled in Melia (2017). Power-law cosmologies with 
$n \geq 1$ have been explored against data in several works such as Gehlaut et al. (2003), Dev et al. (2008), Sethi et al. (2005), Zhu et al. (2008), Shafer (2015), Rani et al. (2015), Dolgov et al. (2014), finding $n \sim 1.5$ consistently. In a more recent work (Shafer 2015), power-law and $R_{\mathrm{h}}=c t$ models were tested with SN Ia and BAO datasets and were found to be highly disfavoured against $\Lambda C D M$. As most of these works have used older data for their analyses, this is a good occasion to revise the constraints using more recent data and hence statistically verify the viability of these models against $\Lambda \mathrm{CDM}$.

The paper is structured as follows. A brief introduction to the models is given in Sect. 2. We describe the data and method used for our joint analysis in Sect. 3. Our results and discussion are given in Sect. 4.

\section{Models}

In this section we briefly describe the standard $\Lambda \mathrm{CDM}$ model, power-law and $R_{\mathrm{h}}=c t$ cosmologies that we tested to asses the late-time acceleration. The dominant components of the $\Lambda \mathrm{CDM}$ model at late times are cold dark matter (CDM), treated as dust, and dark energy (DE) fluid with an EoS parameter $w=-1$. The corresponding Friedmann equation is given by

$H(z)^{2}=H_{0}^{2}\left(\Omega_{\mathrm{m}}(1+z)^{3}+\Omega_{k}(1+z)^{2}+\Omega_{\Lambda}(1+z)^{3(1+w)}\right)$,

where $H_{0}$ is the current expansion rate, while $\Omega_{\mathrm{m}}, \Omega_{\Lambda}$, and $\Omega_{k}$ are the dimensionless density parameters. For the flat $\Lambda \mathrm{CDM}$ model, $\Omega_{\mathrm{m}}=1-\Omega_{\Lambda}$. We tested the extensions of $\Lambda$ CDM model, namely the $k \Lambda C D M$ model, with the constraint $\Omega_{\mathrm{m}}=1-\Omega_{\Lambda}-\Omega_{k}$, and the flat $w \mathrm{CDM}$ model with $w$ as a free parameter. The second Friedmann equation, $\ddot{a} / a=-4 \pi / 3 G \sum_{i} \rho_{i}\left(1+3 w_{i}\right)$, gives us insight into the conditions that need to be satisfied to assess the dynamics of the expansion rate. The criteria for acceleration can be derived as $\Omega_{\mathrm{m}} \leq \Omega_{\Lambda} / 2$ for $k \Lambda \mathrm{CDM}$ and $w \leq-1 /\left(3 \Omega_{\Lambda}\right)$ for $\omega \mathrm{CDM}$. We can asses the evidence for acceleration by estimating the confidence level with which the criteria are satisfied.

In a flat power-law cosmological model the scale factor evolves in time as $a(t) \propto t^{n}$, with the Hubble equation $H(z)=$ $H_{0}(1+z)^{1 / n}$. Here, $n>1$ implies an accelerated scenario. Although motivated physically with a total EoS parameter $w_{\text {tot }}=$ $-1 / 3$, the flat $R_{\mathrm{h}}=c t$ model coincides with the power-law model for $n=1$. It is worthwhile noting that Eq. (1) reduces to the functional form of a power-law model for selected parameter values: $\Omega_{\mathrm{m}}=0, \Omega_{\Lambda}=1$ and

$w=\frac{2-3 n}{3 n}$.

The luminosity distance for all these models with their corresponding $H(z)$ is written as

$D_{\mathrm{L}}(z) \equiv \begin{cases}(1+z) c\left(\int_{0}^{z} \frac{\mathrm{d} \xi}{H(\xi)}\right) & \text { for } \Omega_{k}=0 \\ \frac{(1+z) c}{H_{0} \sqrt{-\Omega_{k}}} \sin \left(\sqrt{-\Omega_{k}} H_{0} \int_{0}^{z} \frac{\mathrm{d} \xi}{H(\xi)}\right) & \text { for } \Omega_{k} \neq 0 .\end{cases}$

The theoretical distance modulus is defined as $\mu_{\text {th }}=$ $5 \log \left[D_{\mathrm{L}}(\mathrm{Mpc})\right]+25$. The angular diameter distance is $D_{\mathrm{A}}(z)=$ $D_{\mathrm{L}}(z) /(1+z)^{2}$, which is used in the modelling of BAO data.

\section{Data and method}

We tested the models described in the Sect. 2 against data in the redshift range $0<z \lesssim 8$. We used the observables $\mathrm{SN}$ Ia, BAO,
OHD, and GRB, which are uncorrelated. We performed a joint analysis using all the datasets together by defining a combined likelihood function. We kept the description of the data to a minimum as we refer to Luković et al. (2016) for most of it.

We used the JLA dataset (Betoule et al. 2014) consisting of $740 \mathrm{SN}$, which provides an empirical correction to the absolute magnitude,

$M_{\mathrm{B}}^{\mathrm{corr}}=M_{\mathrm{B}}-\alpha s+\beta c$.

In the statistical method we implemented (Nielsen et al. 2016), the stretch $s$, colour $c$ corrections, and the absolute magnitude $M_{\mathrm{B}}$ are all considered as random Gaussian variables without any redshift dependence. This assumption does not account for the selection effects in $s$ and $c$ corrections. The JLA dataset has been corrected for the selection bias only in the apparent magnitude (Betoule et al. 2014), which is why the correction in $s$ and $c$ have to be explicitly included when they are modelled as distributions. As described in the introduction, we used the less flexible modelling of Nielsen et al. (2016) to show that even in this case the evidence for acceleration is very strong. Different methods for treating the selection bias in the SN data and their shortcomings have been discussed in Kessler \& Scolnic (2017). It is of high importance to study these effects, which we will address in a forthcoming paper. The SN Ia likelihood $\mathcal{L}_{\mathrm{SN}}$ used here is described in Nielsen et al. (2016), Shariff et al. (2016), Luković et al. (2016).

The BAO data are available for the compound observable $D_{\mathrm{V}}$ defined in Eisenstein et al. (2005),

$D_{\mathrm{V}}(z)=\left[(1+z)^{2} D_{\mathrm{A}}^{2}(z) \frac{c z}{H(z)}\right]^{1 / 3}$.

It is important to note that the observable $D_{\mathrm{V}}$ is usually presented as a ratio with $r_{\mathrm{d}}$, the sound horizon at the drag epoch. For the purpose of the model selection, we fit $r_{\mathrm{d}}$ as a free parameter instead of using the standard fit function for the drag epoch $z_{\mathrm{d}}$ (Eisenstein et al. 1998), as it is not very straightforward to use it for the power-law cosmologies. A similar approach was also implemented in Shafer (2015). Hence, the parameters $H_{0}$ and $r_{\mathrm{d}}$ are now degenerate, and BAO data by itself are only able to constrain the combination $r_{\mathrm{d}} \times H_{0}$ and $\Omega_{\mathrm{m}}$. To avoid correlations among different BAO data points, we used only six measurements taken from Beutler et al. (2011), Anderson et al. (2014), Ross et al. (2015), Delubac et al. (2015), Font-Ribera et al. (2014), also summarised in Table 1 of Luković et al. (2016). A simple likelihood for the uncorrelated data was then implemented as

$\mathcal{L}_{\mathrm{BAO}} \propto \exp \left[-\frac{1}{2} \sum_{i=1}^{6}\left(\frac{r_{\mathrm{d}} / D_{\mathrm{V}}^{i}-r_{\mathrm{d}} / D_{\mathrm{V}}\left(z_{i}\right)}{\sigma_{r_{\mathrm{d}} / D_{\mathrm{V}}}^{i}}\right)^{2}\right]$.

The measurements of the expansion rate were estimated using the differential age (DA) method suggested in Jimenez \& Loeb (2002), which considers pairs of passively evolving red galaxies at similar redshifts to obtain $\mathrm{d} z / \mathrm{d} t$. We used a compilation of 30 uncorrelated DA points taken from Simon et al. (2005), Stern et al. (2010), Moresco et al. (2012a), Moresco et al. (2016), Moresco (2015), Zhang et al. (2014) obtained using BC03 models. We implemented a simple likelihood function assuming all the data are uncorrelated.

$\mathcal{L}_{\mathrm{OHD}} \propto \exp \left[-\frac{1}{2} \sum_{i=1}^{30}\left(\frac{H_{i}-H\left(z_{i}\right)}{\sigma_{H_{i}}}\right)^{2}\right]$. 
Finally, we used the GRB dataset comprising of 109 observations compiled with the well-known Amati relation (Amati et al. 2002, 2008, 2009). The dataset has 50 GRBs at $z<1.4$ and 59 GRBs at $z>1.4$, in a total range of $0.1<z<8.1$. The dataset is given in Tables 1 and 2 of Wei (2010). The distance modulus $\mu_{\mathrm{GRB}}$ and the corresponding standard deviation can be defined as

$$
\begin{aligned}
\mu_{\mathrm{GRB}} & =\frac{5}{2}\left(\log _{10}\left[\frac{(1+z)}{4 \pi}\left(\frac{E_{p, i}}{300 \mathrm{keV}}\right)^{b} \frac{S_{\text {bolo }}^{-1}}{100 \mathrm{pc}^{2}}\right]+\lambda\right) \\
\sigma_{\mu_{\mathrm{GRB}}} & =\left(\frac{5}{2 \log (10)}\right)^{2}\left[\left(\frac{b \sigma_{E_{p, i}}}{E_{p, i}}\right)^{2}+\left(\frac{\sigma_{S_{\text {bolo }}}}{S_{\text {bolo }}}\right)^{2}+\sigma_{\text {sys }}^{2}\right] .
\end{aligned}
$$

We adopted $\sigma_{\text {sys }}=0.7571$, following the model-independent calibration in Feng \& Li (2016). The likelihood for the GRB is defined as

$$
\mathcal{L}_{\mathrm{GRB}} \propto \exp \left[-\frac{1}{2} \sum_{i=1}^{109}\left(\frac{\mu_{\mathrm{GRB}}^{i}-\mu_{\mathrm{th}}^{i}}{\sigma_{\mu_{\mathrm{GRB}}}^{i}}\right)^{2}\right] .
$$

The joint likelihood for these four independent observables is given as $\mathcal{L}_{\text {tot }}=\mathcal{L}_{\mathrm{SN}} \mathcal{L}_{\mathrm{OHD}} \mathcal{L}_{\mathrm{BAO}} \mathcal{L}_{\mathrm{GRB}}$. We used the two most common criteria for a model comparison in cosmology, namely the Akaike information criteria (AIC; Akaike 1974) and the Bayesian information criteria (BIC; Schwarz et al. 1978). The AIC and BIC values for large number of measurements are defined as

$$
\begin{aligned}
& \mathrm{AIC}=-2 \log \mathcal{L}^{\max }+2 N_{\mathrm{p}}, \\
& \mathrm{BIC}=-2 \log \mathcal{L}^{\max }+N_{\mathrm{p}} \log \left(N_{\text {data }}\right),
\end{aligned}
$$

where $N_{\mathrm{p}}$ and $N_{\text {data }}$ are the number of parameters and data points. The $\Delta(\mathrm{AIC})=\mathrm{AIC}-\mathrm{AIC}_{\text {ref }}$ criterion takes into account the number of parameters to estimate the amount of information lost in one model when compared to a reference model, in our case, $\Lambda C D M$. We define the $\Delta(\mathrm{BIC})$ as similar to $\Delta(\mathrm{AIC})$. A negative value of the $\Delta(\mathrm{AIC})$ or $\Delta(\mathrm{BIC})$ indicates that the model in comparison performs better than the reference model.

\section{Results and discussion}

In this section we present the results obtained from our joint analysis for the models and data given in the earlier sections. We first present our assessment for the current accelerated state of the Universe and then comment on the model comparison using the AIC and BIC statistics.

The SN Ia Hubble diagram was claimed to be consistent with a uniform rate of expansion in Nielsen et al. (2016) as the analysis in $k \Lambda C D M$ model differs from the non-accelerating criterion by only $\lesssim 3 \sigma$. We reproduce this result and agree with this statement (see top panel of Fig. A.1). However, there is a strong prejudice for a flat Universe from the CMB data (Ade et al. 2016), and hence it is important to analyse SN Ia and other cosmological data in the context of a flat $w \mathrm{CDM}$ model. We find that the evidence for acceleration in the $\Omega_{\mathrm{m}}-w$ plane is much more significant $(\geq 4.56 \sigma)$ than the marginal $(\geq 2.88 \sigma)$ found in the $\Omega_{\mathrm{m}}-\Omega_{\Lambda}$ plane (see Fig. A.1). The claimed marginal evidence for acceleration that corresponds to a very low matter density becomes more significant in both $k \Lambda C D M$ and $w C D M$ models when more physical values of $\Omega_{\mathrm{m}}$ are considered. In Fig. A.1 contours for the best-fit regions of the supernova dataset and our joint analysis are shown. The joint analysis improves the evidence for acceleration in the $k \Lambda \mathrm{CDM}$ model to $4.98 \sigma$ and in $\omega \mathrm{CDM}$ model to $5.38 \sigma$.
Table 1. Best-fit parameters for the joint analysis of $\mathrm{SN}+\mathrm{BAO}+$ $\mathrm{DA}+\mathrm{GRB}$ datasets, with $1 \sigma$ errors.

\begin{tabular}{ccccc}
\hline \hline Model & $H_{0}\left[\mathrm{~km} \mathrm{~s}^{-1} \mathrm{Mpc}^{-1}\right]$ & $n$ & $\Omega_{\mathrm{m}}$ & $r_{\mathrm{d}}[\mathrm{Mpc}]$ \\
\hline$R_{\mathrm{h}}=c t$ & $62.4 \pm 1.4$ & 1. & - & $148.3 \pm 3.6$ \\
Power-law & $64.2 \pm 1.7$ & $1.08 \pm 0.04$ & - & $147.0 \pm 3.6$ \\
$\Lambda$ CDM & $66.4 \pm 1.8$ & - & $0.361 \pm 0.023$ & $148.6 \pm 3.7$ \\
\hline
\end{tabular}

Notes. We do not quote the parameters of SN and GRB models, which are considered as nuisance parameters.

The parameter space $\Omega_{\mathrm{m}}-w$ allows us to explore points that correspond to the functional forms of $R_{\mathrm{h}}=c t$ and powerlaw models for specific values of the parameters in $w \mathrm{CDM}$. The point $\left(\Omega_{\mathrm{m}}, w\right)=(0,-1 / 3)$, shown in red in the bottom panel of Fig. A.1, phenomenologically reproduces the expansion law of the $R_{\mathrm{h}}=c t$ model (cf. Eq. (1)). Similarly, the point $\left(\Omega_{\mathrm{m}}, w\right)=(0,-0.38)$ corresponds to the best-fit value of $n=1.08$ in the power-law model from our joint analysis (cf. Table 1 and Eq. (2)). Using SN data alone, the best-fit model for the power-law cosmology is found to be $n=1.28$ (corresponding to $w=-0.48$ ), implying an accelerating Universe (cf. Sect. 2). In the $\Omega_{\mathrm{m}}-w$ plane this point lies within the $2 \sigma \mathrm{SN}$ confidence region, in contrast to the $R_{\mathrm{h}}=c t$ at $4.56 \sigma$. However, when the joint analysis is performed, these points are at $5 \sigma$ and $5.5 \sigma$ for the power-law and $R_{\mathrm{h}}=c t$ models, respectively.

The best-fit parameter values for the models considered in this work are presented in Table 1 . The values for the fitted $r_{\mathrm{d}}$ parameter are consistent with the estimates (see Ade et al. 2016) using the fit function for the drag epoch from Eisenstein et al. (1998). In our analysis, the best-fit value for the index $n$ is driven towards unity. This result is quite different form the previous $n$ estimates (Dolgov et al. 2014; Shafer 2015; Rani et al. 2015), which consistently suggest $n \sim 1.5$. The modification in the statistical method for the SN analysis enables this change. While the value we find for BAO data alone $(n=0.94)$ is consistent with the value given by Shafer (2015) $(n=0.93)$, for BAO+SN data we find $n=1.11$ in contrast to his $n=1.52$. Our best-fit value for the joint analysis $(n=1.08 \pm 0.02)$ is now consistent with $n=1.14 \pm 0.05$ found by Zhu et al. (2008) using X-ray cluster data.

It is clear that the $H_{0}$ estimates for the power-law and $R_{\mathrm{h}}=c t$ models are very different from the direct estimate in Riess et al. (2016), which is a well-established problem for $\Lambda$ CDM (Luković et al. 2016; Bernal et al. 2016). In this work, the joint analysis provides $H_{0}=66.4 \pm 1.8 \mathrm{~km} \mathrm{~s}^{-1} \mathrm{Mpc}^{-1}$ for the $\Lambda \mathrm{CDM}$ scenario. We note that this value is consistent with our previous estimate (Luković et al. 2016), but with a higher error due to the difference in the BAO analysis (see Sect. 3). In any case, this value still remains different from the direct estimate at $2.7 \sigma$.

We stress that the Milne model with $\Omega_{\mathrm{m}}=\Omega_{\Lambda}=0$ and $\Omega_{k}=1$ does not correspond to the flat $R_{\mathrm{h}}=c t$ model. In fact, these two models share the same Hubble equation and $\operatorname{EoS}(\rho+$ $3 p=0$ ), but do not have the same $D_{\mathrm{L}}$ as the negative curvature in the Milne model corresponds to $D_{\mathrm{L}} \propto(1+z) \sinh (\log (1+$ z)) with $\Omega_{k}=1$, whereas in the $R_{\mathrm{h}}=c t$ model $D_{\mathrm{L}} \propto(1+$ $z) \log (1+z)$. In the SN Ia Hubble diagram it is difficult to see any significant difference among the $\Lambda \mathrm{CDM}$, Milne, and $R_{\mathrm{h}}=c t$ model predictions, but their performance can be more effectively tested with the information criteria.

The analysis of SN Union 2.1 data described by Melia (2012a) calls for a non-accelerating scenario, as the $R_{\mathrm{h}}=c t$ model was claimed to perform on par with $\Lambda$ CDM. This point 
Table 2. $\Delta$ (AIC) and $\Delta$ (BIC) comparisons for models with $\Lambda \mathrm{CDM}$ as the reference.

\begin{tabular}{ccccc}
\hline \hline & $\Delta(\mathrm{AIC})_{\text {Joint }}$ & $\Delta(\mathrm{AIC})_{\mathrm{SN}}$ & $\Delta(\mathrm{BIC})_{\text {Joint }}$ & $\Delta(\mathrm{BIC})_{\mathrm{SN}}$ \\
\hline Power-law & 28.02 & 2.0 & 28.02 & 2.0 \\
$R_{\mathrm{h}}=c t$ & 30.83 & 21.79 & 26.05 & 17.20 \\
Milne & 66.39 & 9.78 & 61.62 & 5.19 \\
\hline
\end{tabular}

Notes. "Joint" corresponds to the joint analysis with $\mathrm{SN}+\mathrm{BAO}+$ $\mathrm{DA}+\mathrm{GRB}$ datasets.

was also taken by Nielsen et al. (2016), who analysed the JLA dataset with their improved statistical method. In our work, using the same technique for analysing the JLA data, we find that $R_{\mathrm{h}}=c t$ performs poorly when compared to $\Lambda \mathrm{CDM}$ with $\Delta(\mathrm{AIC})_{\mathrm{SN}} \sim 22$, while a power-law model is performing as well as $\Lambda \mathrm{CDM}$ with $n \sim 1.28$ from $\mathrm{SN}$ data. Our results are consistent with the previous work by Shafer (2015). The values of $\Delta(\mathrm{AIC})_{\mathrm{SN}}$ obtained from the SN data alone are shown in Table 2.

We note that the Milne model was claimed to perform marginally worse than $\Lambda \mathrm{CDM}$ using the $\mathrm{SN}$ data alone (Nielsen et al. 2016). In our work, we find for this model $\Delta(\mathrm{AIC})_{\mathrm{SN}}=9.8$ high enough to reject a model. The Milne model fails to keep up when the high redshift GRB $\left(\Delta(\mathrm{AIC})_{\mathrm{GRB}} \sim 20\right)$ and $\mathrm{BAO}\left(\triangle(\mathrm{AIC})_{\mathrm{BAO}} \sim 38\right)$ data are used, yielding a total $\Delta(\mathrm{AIC})_{\text {Joint }} \sim 66.4$ (see Table 2$)$. The AIC statistics disfavours the power-law models by $\Delta\left((\mathrm{AIC})_{\text {Joint }}\right) \sim 28$ and the $R_{\mathrm{h}}=c t$ model by $\Delta\left((\mathrm{AIC})_{\text {Joint }}\right) \sim 30$ in comparison to the $\Lambda \mathrm{CDM}$ model. Our joint analysis shows that all the three models (Milne, powerlaw and $R_{\mathrm{h}}=c t$ ) are strongly disfavoured with respect to $\Lambda \mathrm{CDM}$ (Table 2).

\section{Conclusions}

Contrary to the claim by Nielsen et al. (2016), we find that the SN data alone indicate an accelerating Universe at more than $4.56 \sigma$ confidence level. This evidence becomes even stronger $(5.38 \sigma)$ when we perform the joint analysis combining $\mathrm{SN}$, $\mathrm{BAO}, \mathrm{OHD}$, and GRB data. The non-accelerating $R_{\mathrm{h}}=c t$ model at once fails to explain these data, resulting in $\Delta$ (AIC) $)_{\text {Joint }} \sim 30$ with respect to $\Lambda \mathrm{CDM}$. Although the power-law model performs slightly better that the $R_{\mathrm{h}}=c t$ model, it similarly fails with a $\Delta$ (AIC $)_{\text {Joint }} \sim 28$. Our analysis shows that the possibility of models with a uniform expansion rate is excluded given the current low-redshift data. In conclusion, on one hand we re-assert that the current expansion of our Universe is accelerated, and on the other hand, that $\Lambda \mathrm{CDM}$ still constitutes the base line for a concordance model in cosmology.

\section{References}

Ade, P., Aghanim, N., Arnaud, M., et al. 2016, A\&A, 594, A13 Akaike, H. 1974, IEEE Transactions on Automatic Control, 19, 716
Amati, L., Frontera, F., Tavani, M., et al. 2002, A\&A, 390, 81 Amati, L., Guidorzi, C., Frontera, F., et al. 2008, MNRAS, 391, 577 Amati, L., Frontera, F., \& Guidorzi, C. 2009, A\&A, 508, 173 Anderson, L., Aubourg, É., Bailey, S., et al. 2014, MNRAS, 441, 24 Bernal, J. L., Verde, L., \& Riess, A. G. 2016, J. Cosmol. Astropart. Phys., 2016, 019

Betoule, M., Kessler, R., Guy, J., et al. 2014, A\&A, 568, A22

Beutler, F., Blake, C., Colless, M., et al. 2011, MNRAS, 416, 3017

Bikwa, O., Melia, F., \& Shevchuk, A. 2012, MNRAS, 421, 3356

Bilicki, M., \& Seikel, M. 2012, MNRAS, 425, 1664

Delubac, T., Bautista, J. E., Busca, N. G., et al. 2015, A\&A, 574, A59

Dev, A., Sethi, M., \& Lohiya, D. 2001, Phys. Lett. B, 504, 207

Dev, A., Safonova, M., Jain, D., \& Lohiya, D. 2002, Phys. Lett. B, 548, 12

Dev, A., Jain, D., \& Lohiya, D. 2008, ArXiv e-prints [arXiv: 0804. 3491] Dolgov, A. D. 1997, Phys. Rev. D, 55, 5881

Dolgov, A., Halenka, V., \& Tkachev, I. 2014, J. Cosmol. Astropart. Phys., 10, 047

Eisenstein, D. J., Hu, W., \& Tegmark, M. 1998, ApJ, 504, L57

Eisenstein, D. J., Zehavi, I., Hogg, D. W., et al. 2005, ApJ, 633, 560

Feng, C.-J., \& Li, X.-Z. 2016, ApJ, 821, 30

Font-Ribera, A., Kirkby, D., Busca, N., et al. 2014, J. Cosmol. Astropart. Phys., 5,27

Gehlaut, S., Kumar, P., Geetanjali, \& Lohiya, D. 2003, ArXiv e-prints [arXiv: astro-ph/0306448]

Jimenez, R., \& Loeb, A. 2002, ApJ, 573, 37

Kessler, R., \& Scolnic, D. 2017, ApJ, 836, 56

Kumar, R. 2016, J. Mod. Phys. D, submitted [arXiv: 1611.03728]

Lewis, G. F. 2013, MNRAS, 432, 2324

Lewis, G. F., \& van Oirschot, P. 2012, MNRAS, 423, L26

Luković, V. V., D’Agostino, R., \& Vittorio, N. 2016, A\&A, 595, A109

Melia, F. 2012a, AJ, 144, 110

Melia, F. 2012b, ArXiv e-prints [arXiv: 1205.2713]

Melia, F. 2015, MNRAS, 446, 1191

Melia, F. 2017, MNRAS, 464, 1966

Melia, F., \& Shevchuk, A. S. H. 2012, MNRAS, 419, 2579

Mitra, A. 2014, New Astron., 30, 46

Moresco, M. 2015, MNRAS, 450, L16

Moresco, M., Cimatti, A., Jimenez, R., et al. 2012a, J. Cosmol. Astropart. Phys., 8, 006

Moresco, M., Verde, L., Pozzetti, L., Jimenez, R., \& Cimatti, A. 2012b, J. Cosmol. Astropart. Phys., 7, 053

Moresco, M., Pozzetti, L., Cimatti, A., et al. 2016, J. Cosmol. Astropart. Phys., 05,014

Nielsen, J. T., Guffanti, A., \& Sarkar, S. 2016, Sci. Rep., 6, 35596

Perlmutter, S., Aldering, G., Goldhaber, G., et al. 1999, ApJ, 517, 565

Rani, S., Altaibayeva, A., Shahalam, M., Singh, J. K., \& Myrzakulov, R. 2015, J. Cosmol. Astropart. Phys., 3, 031

Riess, A. G., Filippenko, A. V., Challis, P., et al. 1998, AJ, 116, 1009

Riess, A. G., Macri, L. M., Hoffmann, S. L., et al. 2016, ApJ, 826, 56

Ross, A. J., Samushia, L., Howlett, C., et al. 2015, MNRAS, 449, 835

Rubin, D., \& Hayden, B. 2016, ApJ, 833, L30

Schwarz, G. 1978, The annals of statistics, 6, 461

Sethi, G., Dev, A., \& Jain, D. 2005, Phys. Lett. B, 624, 135

Shafer, D. L. 2015, Phys. Rev. D, 91, 103516

Shariff, H., Jiao, X., Trotta, R., \& van Dyk, D. A. 2016, ApJ, 827, 1

Simon, J., Verde, L., \& Jimenez, R. 2005, Phys. Rev. D, 71, 123001

Stern, D., Jimenez, R., Verde, L., Stanford, S. A., \& Kamionkowski, M. 2010, ApJS, 188, 280

Wei, H. 2010, J. Cosmol. Astropart. Phys., , 1008, 020

Zhang, C., Zhang, H., Yuan, S., et al. 2014, Res. Astron. Astrophys., 14, 1221

Zhu, Z.-H., Hu, M., Alcaniz, J. S., \& Liu, Y.-X. 2008, A\&A, 483, 15 


\section{Appendix A: Additional figure}
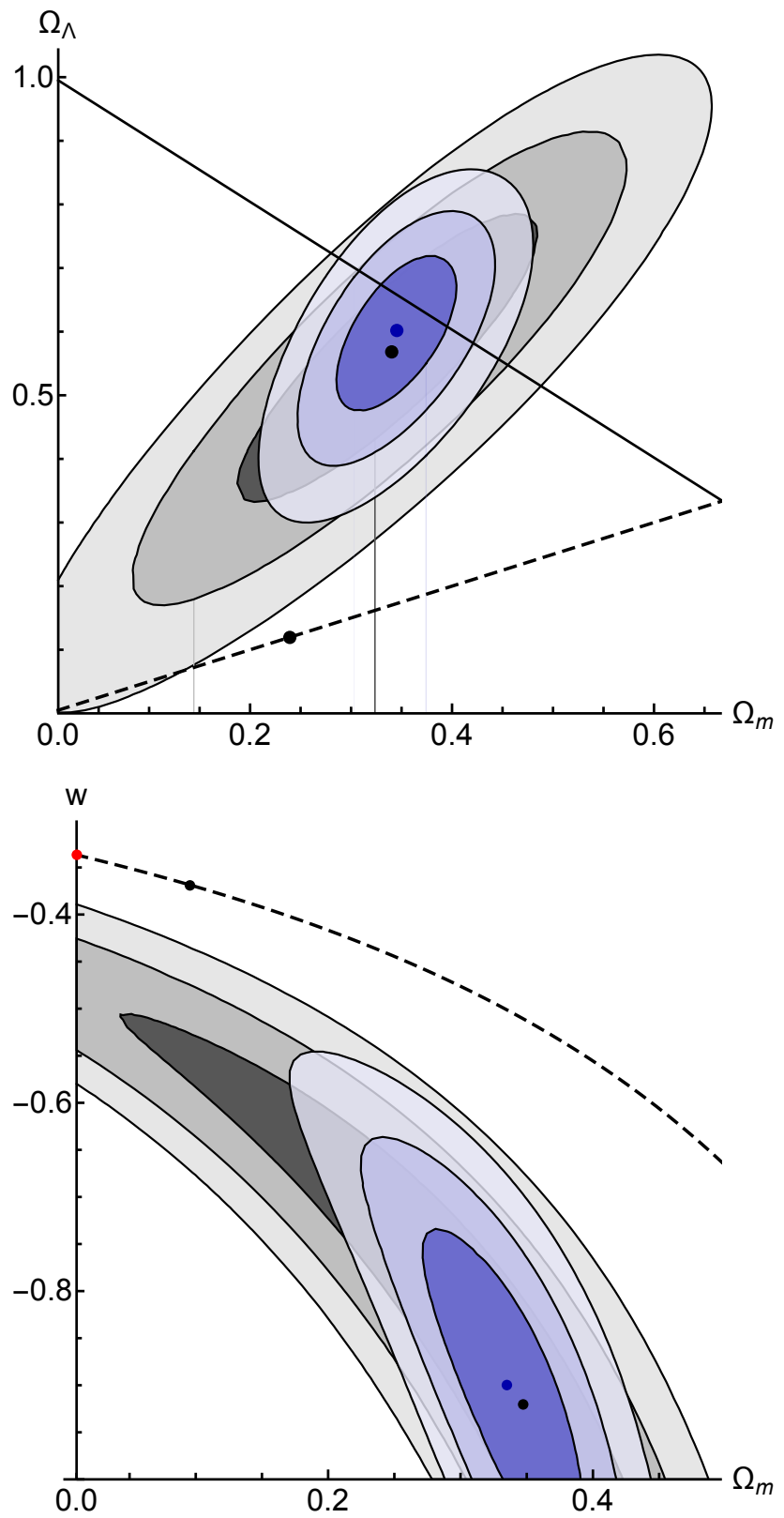

Fig. A.1. Grey and violet confidence regions are obtained from the supernova alone and the joint analysis, respectively. Top panel: 1, 2, and $3 \sigma$ confidence regions in the $\Omega_{\mathrm{m}}-\Omega_{\Lambda}$ parameter space for $k \Lambda \mathrm{CDM}$. The solid line identifies the flat $\Lambda \mathrm{CDM}$ models. The dashed line gives the no-acceleration criterion: $\Omega_{\mathrm{m}}=\Omega_{\Lambda} / 2$. The black point is where the $4.98 \sigma$ confidence contour of the joint likelihood touches the dashed line. Bottom panel: 1,2 , and $3 \sigma$ confidence regions in the $\Omega_{\mathrm{m}}-w$ parameter space for flat $w \mathrm{CDM}$. As in the top panel, the dashed curve identifies the no-acceleration criterion: $w=-1 /\left(3\left(1-\Omega_{\mathrm{m}}\right)\right)$. Likewise, the black point is where the $5.38 \sigma$ confidence contour of the joint likelihood touches the dashed line. 